\title{
Email Overload: Causes, Consequences and the Future
}

\author{
Miloš Vacek
}

\begin{abstract}
Email overload is a term that was defined by scientists to define a state when users are hardly capable of managing all of their incoming messages and consequently their regular work. The first signs of this problem are from 90's and today we can argue whether much has changed to better or worse since then. This paper, again, attempts to describe the problem and unlike other works which concentrate mostly on its consequences studies the causes that should be eliminated. A case study was carried out among employees at University of Hradec Králové to describe the current state and later the results were compared to another dataset obtained from the Enron email corpus. The results suggest that behavior of users has not changed much between years 2001 and 2012 and the same communication patterns still occur and cause many hours being used inefficiently dealing with unwanted emails. The paper concludes with possible future solutions that would help to resolve the problem should these undesired patterns be eliminated.
\end{abstract}

Index Terms-Communication, email, email overload, patterns, enron.

\section{INTRODUCTION}

The problem of email overload has been widely discussed in numerous papers, analyzed by researchers and many of its consequences were described in order to minimize the impacts on information users. Nevertheless, not much attention has been paid to possible solutions that would overcome it from the very beginning - getting as few emails in inbox as possible. To improve this state, the causes were focused in a case study of University of Hradec Králové (UHK). The majority of email users at UHK feel at least some level of email overload while $61 \%$ of the email related work is caused by their own colleagues. This is perhaps the most severe conclusion from a data collection conducted within this research. This paper aims to show that it may not be only the case of UHK employees and that this problem is almost as old as email itself. In the autumn 2012, a quantitative research regarding using work email was carried out among the employees of UHK and the findings are now presented.

The author is aware of the fact that to decide whether the obtained numbers are good or bad, better or worse, some sort of comparison is necessary. Although two different data samples were generated during the last academic year, no significant differences were expected and therefore a completely diverse dataset was sought. The Enron email corpus was after all found very useful and also suitable for academic purposes since it has been widely used to test and

Manuscript received August 30, 2013; revised November 25, 2013. This work was supported by the project INDOP No. CZ.1.07/2.2.00/28.0327.

Miloš Vacek is with University of Hradec Králové (e-mail: milos.vacek@uhk.cz). prove various algorithms, hypotheses and communication patterns. It provided exactly the information needed for comparing the samples and to evaluate what changed between years 2001 and 2012 in email communication.

There are two main objectives that bring some added value to this paper. The first is the state-of-the-art of email communication shown on a case study of UHK that gives a baseline for any future research and continuation of improving this state. The second is a definition of a list of undesired email patterns that are likely to cause the effect known as email overload. Both objectives will be useful to author's following work that intends to lighten the burden of tens of emails that must be dealt with by his colleagues every day.

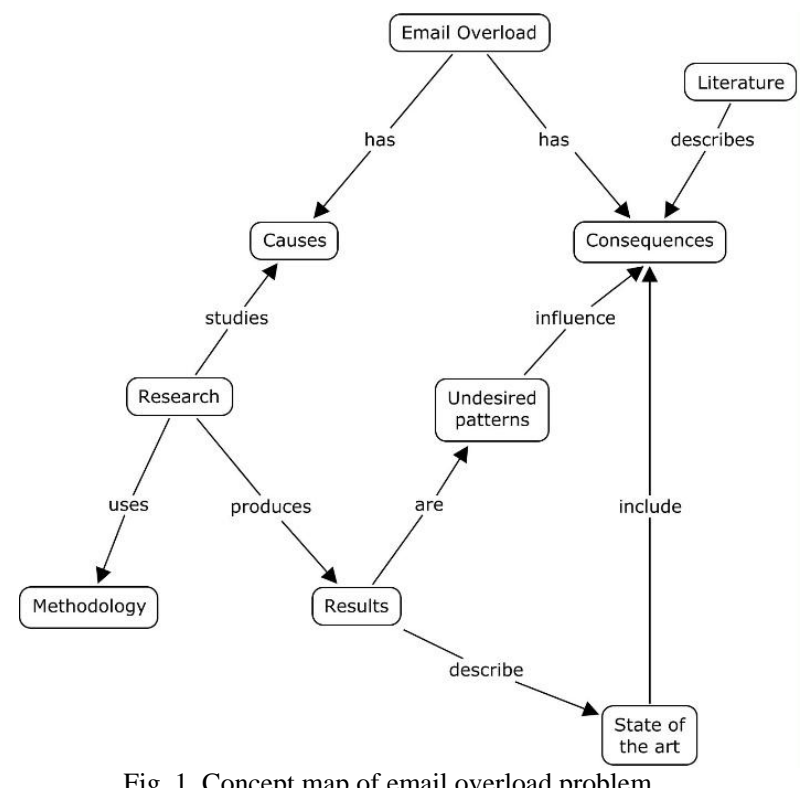

Fig. 1. Concept map of email overload problem.

This paper is structured as suggested on Fig. 1. Email overload problem has Causes and Consequences. The latter are described in the second chapter which gives an overview of previously published works and provides the context for the upcoming sections. Chapter III, Methodology, explains tools and procedures used in research that focused on causes of email overload. The subject of the research is defined in Section IV, Problem definition. The obtained results are described in Section V. Undesired patterns are listed in chapter VI and in the chapter VII the state of the art is discussed as well as some insights in the future. Finally, a summary of the paper is given in VIII.

\section{RELATED WORK}

This chapter gives an overview of literature published on topics related to email overload and email research in general. It includes some recent studies as well as those that 
go back to roots of this problem. The paper widely recognized as the one that originates the term email overload was published in 1996 by Whittaker et al. It described email as a tool that had become used for multiple purposes it had not been designed for, such as document delivery and archiving, work delegation or task tracking. The authors investigated different email management strategies and their conclusions are valuable yet overlooked even today, for example they suggest grouping emails to threads according to the same subject - feature that some email clients still do not support.

It was noticed that since this paper [1] was published the email research has concentrated into three main areas: theoretical and psychological research, practical research which focuses on daily work with email and technical research which designs new features, tools and add-ons. A few examples from each area are cited below.

\section{A. Theoretical and Psychological Research}

The same methodology as in [1] was used 10 years later by other authors in a paper [2] that revisited the email overload problem by analyzing a new email dataset from a technological company. It was found that some metrics, e.g. archive size or number of folders have changed dramatically whereas others, like inbox size, have remained the same. Authors conclude that email overload problem will continue to grow. Another approach was showed in [3] which concerned the number of email recipients - a variable examined in this paper too. Experiments on the Enron corpus revealed that with an increasing number of email recipients the strength of relationships between users decreases. It can be assumed that receiving group emails from someone we do not know even within the same organization can be considered as spamming. To prevent this, companies often deploy policies on maximum number of recipients, using work email for private purposes, etc. This was a subject of study [4] which classified emails without examining its content. The purpose was to create high and low priority email queues (HI priority contains working email, LO contains private) so the email server could deal with requests accordingly, thus more efficiently using the bandwidth. The psychological aspects can be found in [5] where authors predict personality of email writers based on word analysis of emails (e.g. neurotics tend to use more negations) or in [6] where it is claimed that in email communication users often discover their alter ego by behaving more aggressively than in a face to face communication.

\section{B. Practical Research}

Studies in this category often try to propose some solution of a particular problem. This is a case of [7] which introduced three facets of information overload as a large volume of incoming information, inefficient workflow and poor communication quality and conducted training of email users. Then, some hypotheses, such as knowledge of email functions, confirmed that better knowledge after the training support more efficient email communication. The fact that it is important to spread the best practices in using email is also mentioned in [8]. It was claimed that using the best practices has domino effect thus enhancing a better picture of communication and relationships. However, it can be argued that this effect can also work vice versa and bad habits can spread as fast as the good ones, regardless company policies. A different example of practical studies is [9] which focuses on work interruptions caused by checking email too frequently. It verifies and recommends a strategy of creating blocks when employees work on their regular tasks and switching to intervals when incoming emails are dealt with as more efficient. Although the authors propose implementation of this strategy to email clients which would check for incoming emails in predefined intervals the design of such a feature is out scoped.

\section{Technical Research}

The papers in this category introduce real implementations of various features that either enrich user data or make the work with email easier. An example of data enrichment is given in [10] where the described system Acoma gathers context information from various sources based on keywords in machine-generated emails (e.g. account statements, e-tickets, confirmed reservations). The structured information can be used to provide directions to a retrieved address or tracking information for parcel ID. In a different case [11], the email is integrated with social networks (facebook, linkedin, myspace) to find a social context. The practical impact it brings is when a user receives an email from an unknown sender the system automatically retrieves and displays the links based on friend-of-a-friend ontology from social networks. Perhaps a more interesting feature to help email users with better orientation in email workload is presented in [12]. It approaches categorization of emails using own ontology and visualization of email workflow. Thus, if a sender requests some action or delegates a task to colleagues the system generates appropriate user interface to react, e.g. if some approval is demanded the system provides buttons Approve, Reject and Own answer. Also, grouping of emails is possible for better orientation in the context of email workflow.

It seems that all of the works briefly described above as well as many others somehow contribute to solving consequences of email overload. Nevertheless, little work has been published that addresses the causes for this. Why do we have to deal with so much work arising from email? The author believes that the answer lies in the number of incoming emails which has to be decreased to a necessary minimum. One of the likely causes is also described in literature. Hassini [13] in 2004 published a preferred way of communication with students by sending group emails to everybody within each of teacher's courses, thus generating up to 500 emails in a semester! This was 8 years after the first Whittaker's email overload study [1] was published and many e-learning environments with discussion tools were commonly used. However, Hassini still insists on sending email attachments as a way to submit students' works.

This paper focuses on finding the causes for a high number of incoming emails to inboxes and attempts to generalize the findings based on results from the case study of UHK employees. In the following work these causes will be attempted to eliminate. 


\section{Methodology}

This chapter describes the research carried out in order to learn about the email overload problem at UHK. Although the data used in this paper originate at Faculty of Informatics and Management (FIM), another data collection was performed later across all UHK to generalize the result on the whole university and possibly to all affected companies and users. However, results presented in this paper are related to FIM only.

\section{A. FIM Dataset}

FIM is a department of UHK where email is a common tool for communication. Therefore, FIM employees can be referred to as information workers. From informal interviews with employees on various positions it became apparent that email overload problem was present. This finding led to the following data collection and analysis that should confirm a level of severity of the problem.

At first, an updated list of 710 FIM employees and their email addresses was obtained. The list included professors, academics, doctoral students, assistants and other employees. Then, by random selection a sample of 52 employees was chosen and these were contacted by email first and upon agreement a personal interview was conducted. Each participant was introduced to the research objectives and the procedure which included filling up a questionnaire of 10 questions and generating a report from their work email inbox. We used a proprietary piece of Java software (see Fig. 2) where users entered their email credentials and the program attempted to connect the university email server and retrieve following data for specified period from user's inbox:

- Date received

- Sender domain

- Subject

- Number of attachments

- File names

- File sizes

- Message size

- Number of recipients To

- Number of recipients $\mathrm{Cc}$

- Recipients addresses

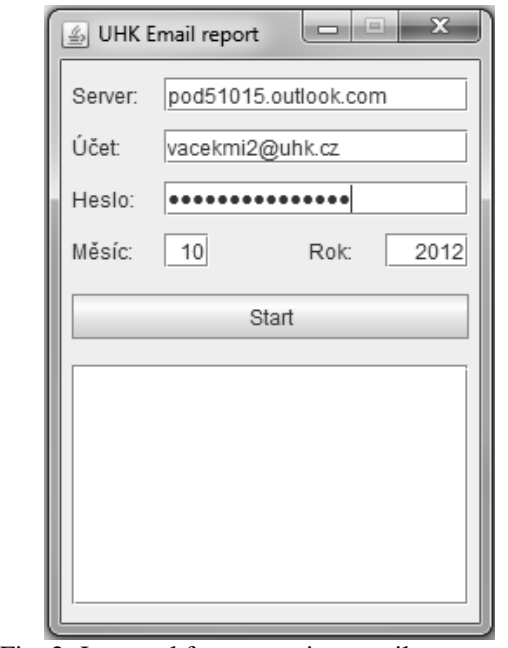

Fig. 2. Java tool for generating email reports.

The program generated a separate flat file for each participant where each email created one line of the report. Only incoming emails from all user folders were summoned and the program filtered emails coming in October 2012. The one-month period in October was chosen deliberately as the first whole month in a semester which should impose a full email workload and a fixed-length period for a comparison with other samples. From the variables above it is clear that no personal data or content was searched though the information retrieved was not anonymous either as an email address from single recipient emails pointed to the inbox owner.

While each report was being generated the participants were answering following questions from the questionnaire:

- Do you consider email as an effective communication tool?

- How many emails do you approximately receive every month?

- What is the percentage of emails that really contain information useful for you?

- How often do you delete emails?

- How many hours a week do you spend by dealing with emails?

- How often do you forget to answer some emails?

- How often do you feel overloaded with email related work?

- What other communication channels do you use?

- Do you use some mobile device to access your email account?

- Can you imagine another technology that would supplement email to you?

Afterwards, another proprietary piece of software was used to import the gathered data from flat files into a relational database. For this purpose a MySQL database instance was created with the structure on Fig. 4. Each email, thus, can reference zero or more attachments and one or more recipients, including their addresses. There was also a possibility of duplicated entries when the same email had been received by two users from the same sample. Each email was therefore tested on uniqueness and the value of column emailCount was increased if such a case was spotted. The algorithm for parsing and saving emails was following (Fig. 3):

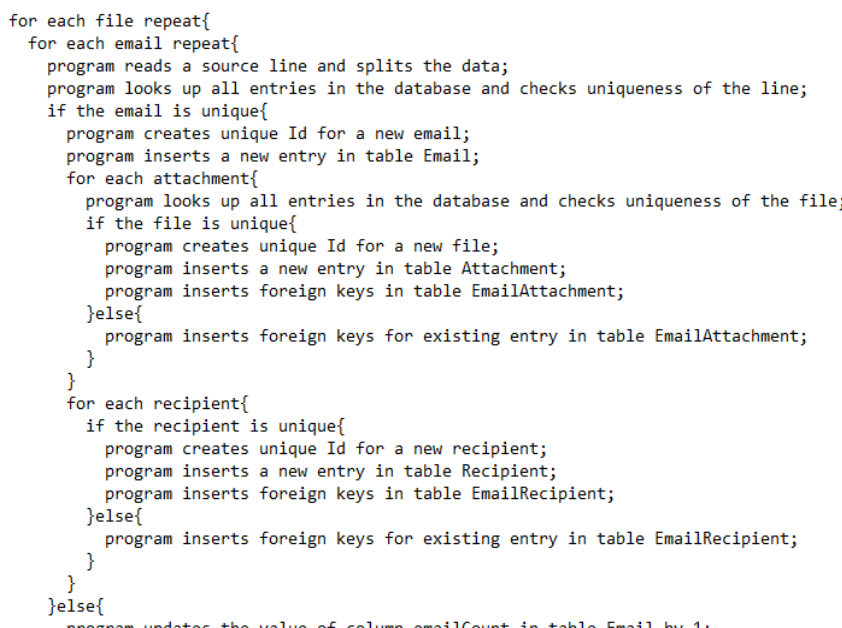

Fig. 3. Pseudo-code of algorithm for transferring data into database. 
This way of saving the data was very useful for following analyses. No matter what problem domain was examined the input data could be easily retrieved by SQL queries.

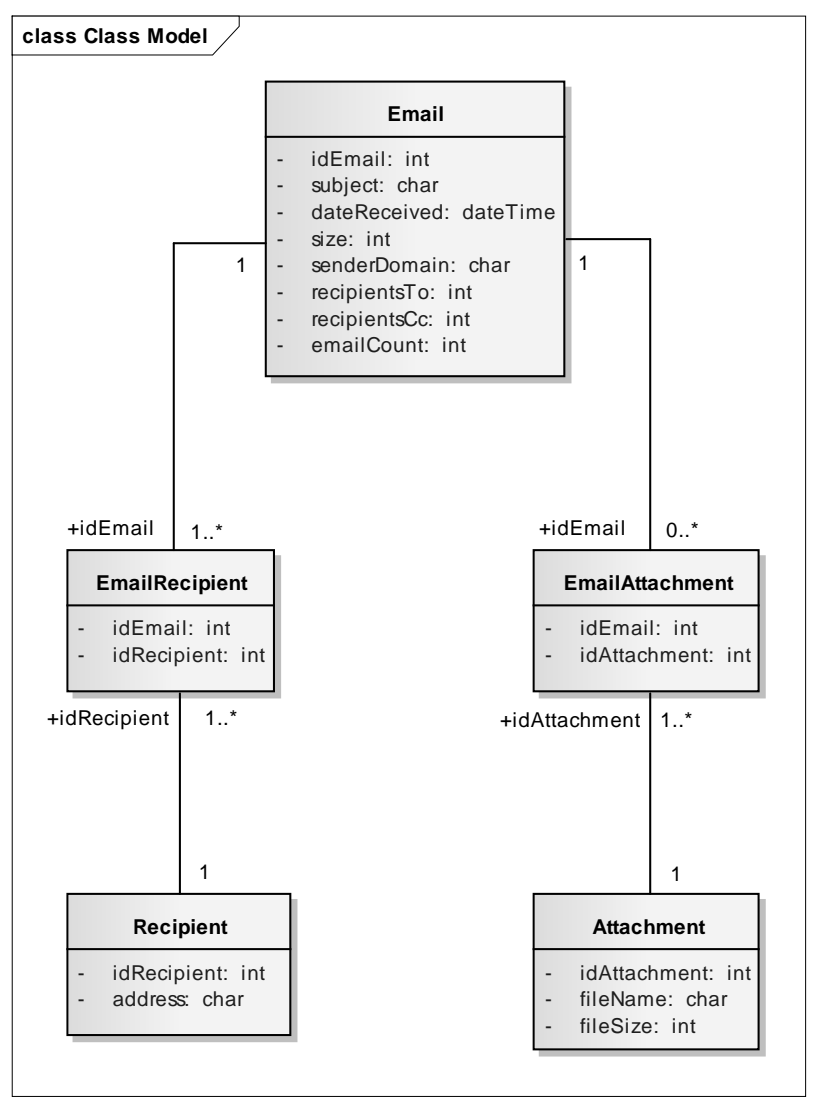

Fig. 4. Database schema.

\section{B. Enron Dataset}

It was mentioned above that to be able to set a baseline for the tested sample from UHK a comparison with a significantly different sample was essential. It was decided that this sample would be generated from the Enron email corpus. There were several reason that led to this decision. The Enron corpus was not only a subject to numerous and very diverse researches [3], [14], [15] but it also provides a snapshot of email communication that was usual among users more than a decade ago. Enron Corporation was an American energy company which left after its bankruptcy an extensive file of complete email communication that was later published for scientific purposes. The archive currently contains about 150 former Enron employees with all of their folders with incoming and outgoing mail counting over 600 000 items from years 1999 to 2002 .

For the purpose of this research the Java tool, used for connecting to the UHK mail server and generating reports, was adjusted to parse the Enron source text files and retrieving the same variables to produce reports with identical structure. The data collection included only incoming email folders and filtered messages received in October 2001. Thus, the two samples are comparable as far as the observed period is concerned. To prepare the Enron sample for a comparison with FIM sample it had to be cut down to count the same number of subject persons. Again, a random selection was made from all Enron employees who produced a non-empty email report (the others might not have been employees in the examined period).
Unfortunately, there were some limitations that had to be dealt with, such as missing attachment files or inconsistent recipient addresses [14]. The produced reports were manually updated with the highest effort to reach the best results and transferred with the other custom tool into a new, empty instance of the database Fig. 1. As a result of this phase, there were two almost identical samples of email data prepared for statistical analyses with a span of 11 years between one and another.

Similarities of the obtained samples were tested on normality using preliminary analyses such as emails received by hours (Fig. 5) which included all emails from the monthly period. These emails were redistributed according to the hour they were received and showed that the numbers of emails received during a day indeed copy working hours.

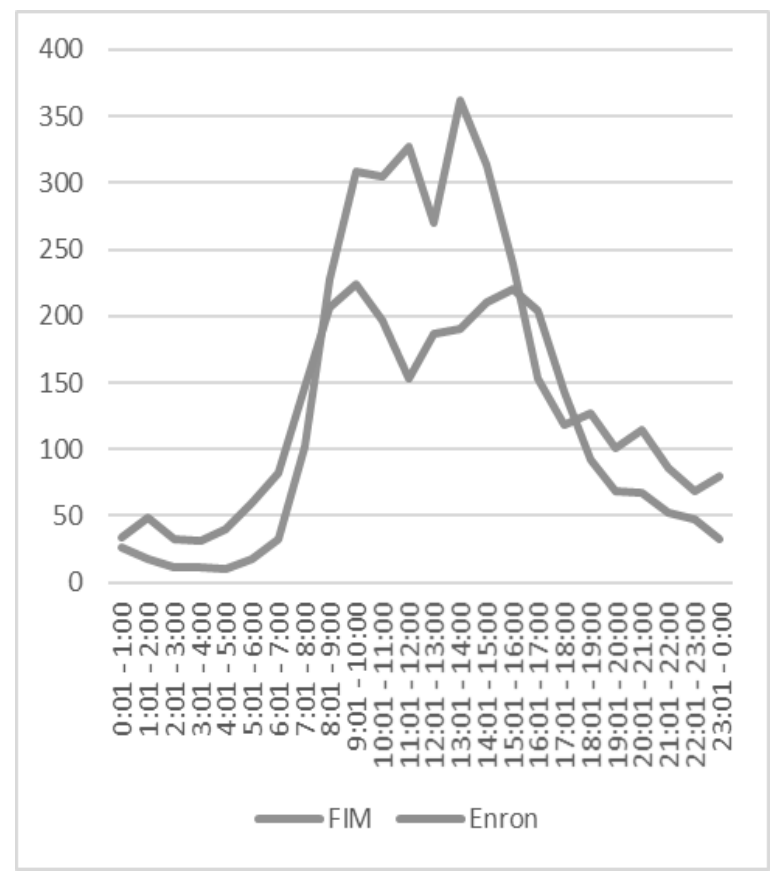

Fig. 5. Emails received by hours.

\section{PRoBlem Definition}

This section describes variables which were tested in the comparison of two samples of email data. Consequently, hypotheses are stated whether there was a significant change in values of these variables between observed samples and years. Later, in Section VI, these variables and their combinations are referred to again as possible factors that cause inboxes filled up with emails.

- Number of emails - it was stated that the more emails users receive the bigger amount of related workload they imply. Therefore it is desired to reduce this number. The total number of messages received in a month by each participant of this research was used.

Hypothesis 1: The total number of incoming messages received by FIM employees in 2012 is greater than number of incoming messages received by Enron employees in 2001.

- Number of attachments - it is assumed that the number of attachments also increase the time users spend by dealing with emails. This assumption is based on the fact that $55 \%$ of all attachments in FIM sample were MS 
Word, Excel and PDF files which are meant to be opened, read and dealt with.

Hypothesis 2: The number of attachments in emails received by FIM employees in 2012 is greater than number of attachments in emails received by Enron employees in 2001.

- Internal emails - it is alarming that email is used mainly for internal communication within one company and often within the same building. It may appear that email is abused in cases when phone call or other form of direct communication would be more appropriate.

Hypothesis 3: The number of internal emails received by FIM employees in 2012 is greater than number of internal emails received by Enron employees in 2001.

- Group emails - as group emails are considered those where number of recipients is greater than 3. This number was set up by the author with an assumption that the relevance of an email content decreases with additional recipients. Simply, one can send some information but is less likely to require some action in response. However, there are other ways of posting information to groups.

Hypothesis 4: The number of group emails received by FIM employees in 2012 is greater than number of group emails received by Enron employees in 2001.

- Number of replies - replies in both samples were identified as messages with subject beginning with RE: Replies are not a particular problem in email communication unless they are combined with internal emails and/or replies cumulating (never ending replies to replies).

Hypothesis 5: The number of replies received by FIM employees in 2012 is greater than number of replies received by Enron employees in 2001.

- Number of forwards - forwarded email were identified as messages with subject beginning with FW: or FWD: Forwarded emails suggest that some recipient was left out from previous communication and often cause confusions about who is the author of the original email (again, especially in chain emails).

Hypothesis 6: The number of forwarded emails received by FIM employees in 2012 is greater than number of forwarded emails received by Enron employees in 2001.

- Number of recipients - although email was not designed as one-to-one communication the increasing number of recipients (which could be abused for spamming) led to policies that set a maximum limit (at UHK this currently 100 recipients of one email). This is also mentioned in [3] that we actually send emails to people we do not know.

Hypothesis 7: The average number of email recipients at FIM in 2012 is greater than average number of email recipients in Enron in 2001.

All the above hypotheses were tested using two independent samples t-tests, based on the source data from each of the two samples, and the interpretation of findings is given.

\section{RESULTS}

This section describes the results of the research from two perspectives. First, it summarizes the process of data collection at FIM, and then it brings an overview of results obtained from statistics calculated for each pair of variable and hypothesis.

TABLE I: BASIC STATISTICS FROM UHK DATASET

\begin{tabular}{lcr}
\hline Variable & Mean & St. Dev. \\
\hline Number of emails & 214,44 & 217,93 \\
Number of attachments & 146,50 & 156,80 \\
Internal emails & 130,69 & 142,60 \\
Group emails & 30,06 & 20,06 \\
Number of replies & 67,19 & 92,74 \\
Number of forwards & 26,19 & 23,91 \\
Avg. number of recipients & 5,06 & 3,22 \\
\hline
\end{tabular}

TABLE II: BASIC STATISTICS FROM ENRON DATASET

\begin{tabular}{lrr} 
Variable & Mean & St. Dev. \\
\hline Number of emails & 173,06 & 202,66 \\
Number of attachments & 12,19 & 12,18 \\
Internal emails & 139,75 & 197,01 \\
Group emails & 93,38 & 166,19 \\
Number of replies & 26,00 & 22,58 \\
Number of forwards & 21,00 & 15,36 \\
Avg. number of recipients & 12,06 & 7,81 \\
\hline
\end{tabular}

TABLE III: RESULTS OF TWO-TAILED T-TEST FOR TWO INDEPENDENT SAMPLES

\begin{tabular}{lrrr}
\hline Variable & df & abs $(\boldsymbol{t}$-value $)$ & $\begin{array}{r}\text { Critical } \\
\text { value }\end{array}$ \\
\hline Number of emails & 30 & 0,56 & 2,04 \\
Number of attachments & $\mathbf{3 0}$ & $\mathbf{3 , 4 2}$ & $\mathbf{2 , 0 4}$ \\
Internal emails & 30 & 0,15 & 2,04 \\
Group emails & 15,44 & 1,51 & 2,13 \\
Number of replies & 30 & 1,73 & 2,04 \\
Number of forwards & 30 & 0,73 & 2,04 \\
Avg. number of recipients & $\mathbf{1 9 , 9 7}$ & $\mathbf{3 , 3 1}$ & $\mathbf{2 , 0 9}$ \\
\hline
\end{tabular}

It was said in (FIM dataset) that 52 email users were randomly chosen for data collection. Unfortunately, not all of them were able to or agreed to participate in the research. Some requests returned back as undeliverable (3), some users refused to participate (5) whereas some did not respond at all (10). From those who responded 6 could not be reached for time reasons (too busy for 20-minute interview). The remaining number of participants were interviewed (28). However, from this number 2 refused to provide data from email inbox for privacy reasons, 3 users did not keep copies of email on the server (empty report was generated) and in 7 cases there was a technical problem with generation. After all, from the theoretical sample of 52 participants there was a return rate of questionnaires 54\% (28) and a return rate of email reports $31 \%$ (16). It means that also 16 non-empty email reports were selected for proceedings from the Enron population. The Table I and Table II summarize basic statistics calculated for each 
sample of email data.

The Table III gives an overview of two-tailed t-tests for statistical significance $\alpha=0.05$.

The numbers can be interpreted in a following way: hypotheses 1, 3, 4, 5 and 6 are rejected, values of examined variables are not greater at FIM in 2012 compared to values from Enron in 2001. Each pair of values is statistically the same. The hypothesis 7 is also rejected with the additional conclusion that the average number of email recipients was greater in Enron in 2001 than at FIM in 2012. The hypothesis 2 is accepted, the number of attachments in emails was significantly higher at FIM in 2012 than in Enron in 2001.

\section{UNDESIRED PATTERNS}

This section lists a number of undesired patterns that have been identified as the possible causes of email overload and which should be avoided in email communication in a company. Further, it is assessed what impacts these patterns may have on email related workload of users. The list is ordered from the most severe ones to the less important ones.

- Internal forwarded emails with attachments sent to many recipients - although this pattern is on the top of the list it does not mean that it occurs rarely. This is a common case of conference "call for papers". When someone receives such an invitation (with additional information in a pdf attachment) they resend it to everybody else no matter if others are interested or not or if the sender knows all recipients. More examples like this could be found.

- Internal or forwarded emails sent to many recipients - even if an email does not contain attachments it is still likely to address people who are not interested. The rule of a limited number of recipients always implies two different situations: there is either someone missing on the list who might be interested or there are people on the list who are not interested.

- Internal emails with attachments - email is not a document management system. It does not support versioning, it has limited storage per user, it creates redundant copies of files for each recipient. It does not support full text search so, for example, information in pdf files is not even searchable.

- All emails sent to many recipients - similarly to forwarded emails, sending emails to a large number of recipients can be treated as spamming and often it fails to obey a company policy (there was a case when an internal email at FIM had 157 recipients, the policy allows 100).

- Internal replies - these emails mean that two colleagues within one company both spent time writing an email and replying to it. Yet emails tend to be abused for this purpose with an excuse that a phone call might be disturbing for the other person.

- All emails with attachments - by attachments here mostly work items are meant and the same as above apply. There are, however, exceptions, for example, images (company logo in email signature) that are also counted as attachments.
Without any evidence given as yet that there are relationships between these patterns that all occur in the FIM data sample (possible causes) and email overload consequences it can be said that there is a huge space for improvement. The following findings were extracted from the questionnaires. Each user at FIM receives on average 242 emails every month. About $29 \%$ of these emails are irrelevant to the recipients. Every user spends more than 8 hours a week by processing emails (reading, writing, answering, etc.) which is above $20 \%$ from normal 40 hours of work. And then, $14 \%$ of email users suffer from email overload on daily basis, $46 \%$ experience it once or twice a week and $71 \%$ of users cannot do their regular work due to processing emails up to three times a month. All interviewed research participants $(100 \%)$ consider email as an effective communication tool and $79 \%$ of them cannot even imagine using something else for communication regardless any possible benefits. That is indeed a huge space for improvement.

\section{DISCUSSION AND FUTURE WORK}

In this chapter there is a summary of the state-of-the-art in email communication at FIM UHK and a few proposals for the future research. There are two possible explanations arising from the conclusion that the volume of incoming emails has not changed since 2001. Either the level of email workload has become stable over years and there is nothing to be afraid of for the future because it will not rise any more. Or Enron users have experienced email overload even in 2001 and this state continues until nowadays. The opinion of Enron employees on this remains, of course, unknown but, considering the facts, that email overload was first described in 1996 [1] and 71\% of FIM email users do feel overloaded from time to time it is much more likely to be the second case. Basically, it could be generalized that anyone who receives regularly more than 10 emails a day might be a victim of email overload.

Participants of this research are not unique in opinion how the current situation could be improved. Most of them admit that emails take too much of their time they would like to spend on other tasks. On the other hand, even bigger proportion do not believe that some other tool, perhaps with more advance features, could help them to deal with communication more efficiently. It has been stated that there are patterns which might be considered as likely causes for email overload yet without evidence. This matter will be observed more closely in the following work. There was another snapshot of email communication at UHK taken in March 2013. Only this time the sample included employees from across the entire university. The new questionnaire had a little more details and the data should enable to discover the possible dependence between the undesired patterns and overflowing email inboxes. The long-term objective is of course a new solution of electronic communication, using efficiently modern IT assets and widely acceptable by end users.

\section{CONCLUSION}

This paper brings an insight into the problem of email 
overload and specifically concerns the current situation at University of Hradec Králové. A high level overview of related literature is given and provides an introduction and context for the research that was carried out in October 2012 among employees of Faculty of Informatics and Management. The quantitative research included a questionnaire and email datasheets generated from inboxes of participants. The data was then analyzed and compared to a similar data snapshot from 2001 retrieved from Enron email corpus. It was discovered that the volume of email communication has not changed significantly. However, on the opposite side stands the fact that the majority of email users suffer from email overload. Some possible causes of this fact have been outlined, in the form of undesired patterns in email communication, and will be analyzed in the following research for which the data collection was gathered in March 2013. Finally, the paper concludes with the actual state of email communication at UHK and suggests some improvements for the future.

\section{REFERENCES}

[1] S. Whittaker and C. Sidner, "Email overload: Exploring personal information management of email," in Proc. the SIGCHI Conference on Human Factors in Computing Systems, 1996, pp. 276 - 283.

[2] D. Fischer, A. J. Brush, E. Gleave, and M. A. Smith, "Revisiting whittaker \& sidner's 'email overload' ten years later," in Proc. the 20th Conference on Computer Supported Cooperative Work, 2006 , pp. $309-312$.

[3] O. Engel, "Clusters, Recipients and reciprocity: Extracting more value from email communication networks," Social and Behavioral Sciences, vol. 10, pp. 172 - 182, 2011.

[4] M. Wang, M. Tsai, S. Jheng, and C. Tang, "Social feature-based enterprise email classification without examining email contents," Journal of Network and Computer Applications, vol. 35, issue 2, pp. $770-777,2012$.

[5] J. Shen, O. Brdiczka, and J. Liu, "Understanding email writers: personality prediction from email messages," Lecture Notes in Computer Science, vol. 7899, pp. 318 - 330, 2013.

[6] P. Gottschalk, "Maturity model for email communication in knowledge organizations: The case of police investigations," International Journal of Law, Crime and Justice, vol. 36, issue 1, pp. $54-66,2008$
[7] R. Soucek and K. Moser, "Coping with information overload in email communication: Evaluation of a training intervention," Computers in Human Behavior, vol. 26, issue 6, pp. 1458 - 1466, 2010.

[8] C. Hu, A. F. L. Wong, H. M. Cheah, and P. Wong, "Patterns of Email Use by Teachers and Implications: A Singapore Experience," Computers \& Education, vol. 53, issue 3, pp. 623 - 631, 2009

[9] A. Gupta, R. Sharda, and R. A. Greve, "You've got email! Does it really matter to process emails now or later?" Information Systems Frontiers, vol. 13, issue 5, pp. 637 - 653, 2011.

[10] M. Laclavik, M. Šeleng, Š. Dlugolinsky, E. Gatial, and L. Hluchý, "Tools for email based recommendation in enterprise," ENTERprise Information Systems, Communications in Computer and Information Science, vol. 109, pp. 209 - 218, 2010.

[11] T. Tran, J. Rowe, and S. F. Wu, "Social email: A framework and application for more socially-aware communications," Social Informatics, Lecture Notes in Computer Science, vol. 6430, pp. 203 215,2010

[12] S. Scerri, "Semantics for Enhanced Email Collaboration," Knowledge Discovery, Knowledge Engineering and Knowledge Management, Communications in Computer and Information Science, vol. 272, pp 413 - 427, 2013.

[13] E. Hassini, "Student-instructor communication: The role of email," Computers \& Education, vol. 47, issue 1, pp. 29 - 40, 2006.

[14] A. Chapanond, M. S. Krishnamoorthy, and B. Yener, "Graph theoretic and spectral analysis of enron email data," Computational \& Mathematical Organization Theory, vol. 11, issue 3, pp. 265 - 281, 2005.

[15] P. Kazienko and K. Musiał, "Mining Personal social features in the community of email users," Lecture Notes in Computer Science, vol 4910, pp. $708-719,2008$.

Miloš Vacek was born in Mladá Boleslav, Czech Republic in 1980 . He Studied at University of Huddersfield, United Kingdom and got BA(Hons.) degree in 2004, Master degree in 2007.

He works as a software analyst/architect for marketing agency Actum in Prague. He is also an external student of the doctoral program at University of Hradec Králové. His Recent publications: Vacek M., Krbálek P.: Semantics of Knowledge Map Visualization, 12th International Conference on Applied Informatics and Communications (AIC '12), Istanbul, Turkey, pp. 196-201, 2012. Vacek M., Krbálek P.: Teleology: A modern approach for knowledge mapping; International Journal of Knowledge-Based and Intelligent Engineering Systems, vol. 17, iss. 2, pp. 137-144, 2012.Vacek M., Krbálek P.: Association between Teleology and Knowledge Mapping; Knowledge Engineering, Machine Learning and Lattice Computing with Applications, Lecture Notes in Computer Science, Vol. 7828, 2013, pp. 143-152, 2012.

He cooperated on a research project concerning Collaborative Knowledge Mapping and published papers on e-Government field. Currently works on his dissertation project concerning email overload. 